\title{
RING finger protein 10 is a potential drug target for diabetic vascular complications
}

\author{
SIYU LI, GUIQUAN YU, WEI HUANG, RUIYU WANG, PENG PU and MING CHEN \\ Department of Cardiology, First Affiliated Hospital of Chongqing Medical University, \\ Chongqing 400016, P.R. China
}

Received August 1, 2018; Accepted February 28, 2019

DOI: $10.3892 / \mathrm{mmr} .2019 .10358$

\begin{abstract}
Vascular remodeling induced by long-term hyperglycaemia is the main pathological process in diabetic vascular complications. Thus, vascular remodeling may be a potential therapeutic target in diabetes mellitus (DM) with macrovascular disease. The present study aimed to investigate the effect of RING finger protein 10 (RNF10) on vascular remodeling under conditions of chronic hyperglycaemia stimulation. We found that overexpression of RNF10 clearly decreased intimal thickness and attenuated vascular remodeling in DM. TUNEL staining showed that apoptosis was clearly inhibited, an effect that may be mediated by decreases in Bcl-2 protein expression. Quantitative analysis demonstrated that overexpression of RNF10 could suppress inflammation by reducing the levels of TNF- $\alpha$, and MCP- 1 mRNA and NF- $\kappa$ B protein. Meanwhile, overexpression of RNF10 prevented vascular smooth muscle cell (VSMC) hyperproliferation through the downregulation of cyclin D1 and CDK4 proteins. Notably, short hairpin RNF10 (shRNF10) greatly aggravated the pathological responses of diabetic vascular remodeling. These outcomes revealed that the differential expression of
\end{abstract}

Correspondence to: Dr Peng Pu or Dr Ming Chen, Department of Cardiology, First Affiliated Hospital of Chongqing Medical University, 1 Youyi Road, Yuzhong, Chongqing 400016, P.R. China E-mail:pp841103@sina.com

E-mail: chenmingcq77@163.com

Abbreviations: DM, diabetes mellitus; RNF10, RING finger protein 10; shRNF10, short hairpin RNF10; VSMCs, vascular smooth muscle cells; UPS, ubiquitin-proteasome system; HF, high fat; IPGTT, intraperitoneal glucose tolerance test; BI, balloon injury; PBS, phosphate-buffered saline; Ad, adenovirus; GFP, green fluorescent protein; qPCR, quantitative polymerase chain reaction; TNF- $\alpha$, tumor necrosis factor $\alpha$; MCP-1, monocyte chemotactic protein 1 ; NF- $\kappa \mathrm{B}$, nuclear factor- $\kappa$-gene binding; TUNEL, terminal deoxynucleotidyl transferase dUTP nick end labeling; H\&E, haematoxylin and eosin; Bax, B-cell CLL/lymphoma 2 associated X protein; Bcl-2, B-cell CLL/lymphoma 2; CDK4, cyclin dependent kinase 4

Key words: RING finger protein 10, vascular remodeling, diabetes, inflammation, hyperproliferation, apoptosis
RNF10 had a completely opposite effect on vascular damage under hyperglycaemia, further displaying the core function of RNF10 in regulating vascular remodeling induced by diabetes. Consequently, RNF10 could be a novel target for the treatment of diabetic vascular complications.

\section{Introduction}

Diabetes mellitus (DM) is a serious public health concern worldwide due to its gradually increasing prevalence (1). Diabetes is also an independent risk factor for many cardiovascular diseases, leading to the increased incidence of vascular remodeling among vascular diabetic complications. The pathogeneses of vascular remodeling induced by vascular smooth muscle cell (VSMC) dysfunction involve excessive inflammation and overactivation of proliferation, accompanied by increased amounts of extracellular matrix secretion, ultimately contributing to the thickening of arterial walls (2-5). Although vascular remodeling in diabetes is known to be associated with the combination of these abnormal pathological processes, the specific mechanism is still unknown.

The ubiquitin-proteasome system (UPS), the major mechanism by which proteins are degraded in cells, can degrade $80-90 \%$ of ubiquitinated proteins related to almost all biological activities in the organism, such as apoptosis, inflammation and cell cycle progression (6,7). The UPS has been considered a potential drug target in diabetes and other cardiovascular diseases $(8,9)$. In the entire UPS, the E3 ubiquitin ligase is the most critical, as it identifies and promotes the degradation of substrate proteins by determining the time and specificity of ubiquitination $(7,10)$. Thus, targeting a proper E3 ubiquitin ligase that participates in the regulation of crucial proteins in vascular remodeling could represent a new strategy for the treatment of diabetic vascular complications.

Ring finger protein 10 (RNF10), located on the long arm of chromosome 12, is a member of the RING finger family of E3 ubiquitin ligases, with the function of recruiting ubiquitin ligase E2 and substrate proteins $(11,12)$. A clinical study demonstrated that RNF10 mRNA expression is significantly enhanced in obese patients and greatly increases the risk of diabetes (13). In our previous study, overexpression of RNF10 was shown to significantly prevent neointimal formation by promoting apoptosis reactions and inhibiting $\mathrm{Bcl}-2$ protein 
activation in metabolic syndrome (14). The aim of the present study was to investigate the effect of RNF10 on vascular remodeling and the main pathological processes in diabetic rats after balloon injury, including inflammation, proliferation and apoptosis responses.

\section{Materials and methods}

Animal models. All animal experiments were approved by the Ethics Committee of Chongqing Medical University (Chongqing, China) and were performed according to the ARRIVE guidelines and the UK Animals (Science Procedures) Act, 1986 (https://assets.publishing.service. gov.uk/government/uploads/system/uploads/attachment_data/ file/116843/aspa-draft-guidance.pdf), and its associated guidelines. All experimental male Sprague-Dawley rats (4 weeks of age) were purchased from the Experimental Animal Centre of Chongqing Medical University (Chongqing, China). After one week of acclimation, the rats were randomly divided into control $(n=12)$ and $\mathrm{DM}(\mathrm{n}=60)$ groups. For a total of 12 weeks, the control group was fed a standard diet that included $13 \%$ fat, $61 \%$ carbohydrates, and $26 \%$ protein, while the DM group was fed a high-fat diet with $60 \%$ fat, $21 \%$ carbohydrates, and $19 \%$ protein. The animal experiment was performed in a temperature-controlled room maintained at $22 \pm 2^{\circ} \mathrm{C}$ and a $12 \mathrm{~h} \mathrm{light/dark} \mathrm{cycle} \mathrm{was} \mathrm{maintained.} \mathrm{Groups} \mathrm{of}$ 5 rats were housed in cages with free access to food and water.

The diabetic model was induced by feeding rats a high-fat diet resulting in hyperglycaemia and insulin resistance. All SD rats were fasted overnight (16-18 h) after receiving a standard diet or a high-fat diet for 12 weeks. Then, blood samples were collected from the retro-orbital plexus into a capillary tube to measure the diabetic indices. Plasma was frozen and stored at $-80^{\circ} \mathrm{C}$ after being separated by centrifugation at $1,000 \mathrm{x} \mathrm{g}$ for $30 \mathrm{~min}$ at $-4^{\circ} \mathrm{C}$. Fasting blood glucose was quantified using a portable blood glucose meter (Sinocare Inc., Changsha, Hunan, China). An intraperitoneal glucose tolerance test (IPGTT) was used to detect insulin resistance and impaired glucose tolerance.

Carotid artery balloon injury (BI) and adenovirus (Ad) infection. Within the DM group on a high-fat (HF) diet, all rats were classified into 5 groups based on whether the balloon injury operation and adenovirus infection occurred. Eventually, all the SD rats were divided into the following 6 groups: i) the control group, ii) the $\mathrm{DM}+$ Sham group, iii) the $\mathrm{DM}+\mathrm{BI}$ group, iv) the $\mathrm{DM}+\mathrm{BI}+\mathrm{Ad}-\mathrm{GFP}$ group, v) the $\mathrm{DM}+\mathrm{BI}+\mathrm{Ad}-\mathrm{RNF} 10$ group, and vi) the DM+BI+Ad-shRNF10 group.

Balloon injury was performed and measured as described previously (15); the balloon catheter (Medtronic Inc., Minneapolis, MN, USA) was introduced from the left external carotid artery into the thoracic aorta. The left common carotid artery was damaged by repeatedly pushing and pulling the inflated balloon three times. After washing the damaged artery with phosphate-buffered saline (PBS), $50 \mu 1$ of adenovirus encoding RNF10, shRNF10 or GFP ( $2 \times 10^{10} \mathrm{pfu} / \mathrm{ml}$, Obio Technology Corp. Ltd., Shanghai, China) was injected into the injured artery to incubate for $30 \mathrm{~min}$. After 14 days, the rats were sacrificed by high-dose injection of sodium pentobarbital $(\geq 90 \mathrm{mg} / \mathrm{kg}$ ), and the carotid arteries were harvested.
Histological and morphometric analysis. To examine the establishment of the vascular remodeling model, the obtained carotid tissue sections were stained using haematoxylin and eosin (H\&E) as previously described (16). The carotid arteries of the rats were fixed in $4 \%$ paraformaldehyde for $24 \mathrm{~h}$ and then embedded in paraffin. Next, the sections were prepared for haematoxylin staining at room temperature for $15 \mathrm{~min}$ and eosin staining at room temperature for $5 \mathrm{~min}$. Finally, the sections were stained with picrosirius red for $30 \mathrm{~min}$ at room temperature. Photomicrographs (x100 magnification) were captured with a routine light microscope (cat. no. 4.10.00, Nikon Co., Ltd., Tokyo, Japan).

Terminal deoxynucleotidyl transferase dUTP nick end labeling (TUNEL) assay. To determine the proportion of apoptotic carotid artery cells in the diabetic rats, a TUNEL assay was performed using an In Situ Cell Death Detection Kit-POD (Roche, Basel, Switzerland). First, carotid tissue sections were sequentially incubated with proteinase $\mathrm{K}$ for $30 \mathrm{~min}$. Then, these sections were incubated with membrane disruption solution (Service Bio Inc., Woburn, MA, USA) for $20 \mathrm{~min}$ at room temperature, followed by incubation with a mixture of reagent 1 (TdT) and reagent 2 (dUTP) from the TUNEL kit for $2 \mathrm{~h}$. Next, the sections were incubated with reagent 3 (converter-POD) for $30 \mathrm{~min}$ after incubation with hydrogen peroxide and ethanol for $15 \mathrm{~min}$. Finally, the sections were mounted and observed under a routine light microscope (at magnification, $\mathrm{x} 400$, cat. no. 4.10.00, Nikon Co., Ltd., Tokyo, Japan) after 3,3'-diaminobenzidine (DAB) (Dako; Agilent Technologies, Inc., Santa Clara, CA, USA) was added. The cells with brown and yellow nuclei were TUNEL-positive cells (17). The apoptosis index $=$ number of apoptosis-positive cells $\mathrm{x}$ total number of intimal cells.

Quantitative polymerase chain reaction $(q P C R)$. Total RNA was isolated from the carotid arteries of rats with TRIzol reagent (Takara Bio Inc., Kusatsu, Japan), and up to $1 \mu \mathrm{g}$ of total RNA was reverse transcribed into cDNA following the manufacturer's instructions (Takara Bio Inc.). The reaction protocol was: Polymerase activation for $10 \mathrm{~min}$ at $95^{\circ} \mathrm{C}$ and then 40 amplification cycles of $60 \mathrm{sec}$ at $60^{\circ} \mathrm{C}$. The cycle threshold values were normalized to the level of $\beta$-actin, and the relative gene expression was measured by the $2^{-\Delta \Delta C a}$ method (18). The primers included those for $\beta$-actin (5'-CCCATCTATGAGGGTTACGC-3' and 5'-TTTAATGTC ACGCACGATTTC-3'), RNF10 (5'-ATTTTAGCAACCAGT CCCGTCG-3' and 5'-CCTCATCCCGTCTTCCACCAT-3'), TNF- $\alpha$ (5'-CTACTGAACTTCGGGGTGATC-3' and 5'-GGT CTGGGCCATAGAACTGA-3') and MCP-1 (5'-GCTGAC CCCAAGAAGGAATG-3' and 5'-GTGCTTGAGGTGGTT GTGGA-3').

Western blot analysis. The common carotid total protein was extracted using $10 \mu \mathrm{l} / \mu \mathrm{g}$ RIPA lysis buffer (Beyotime Institute of Biotechnology, Shanghai, China) and quantified via a BCA Protein Assay Kit (Beyotime Institute of Biotechnology). Protein $(40 \mu \mathrm{g})$ was separated on 10-12\% SDS-polyacrylamide gels (Beyotime Institute of Biotechnology) and transferred to PVDF membranes. The membranes were blocked in $5 \%$ non-fat dry milk for approximately $2 \mathrm{~h}$ and incubated 

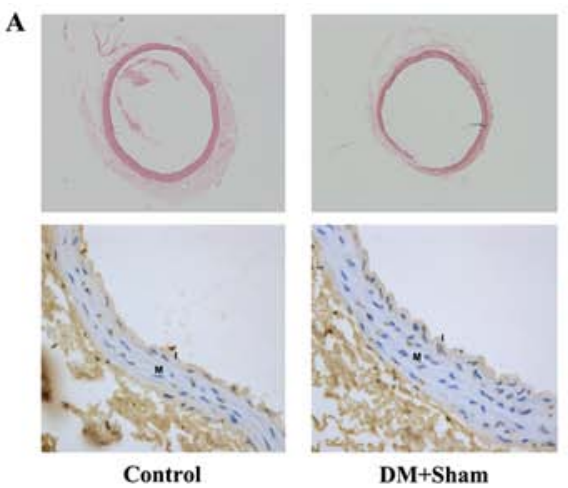

DM+Sham
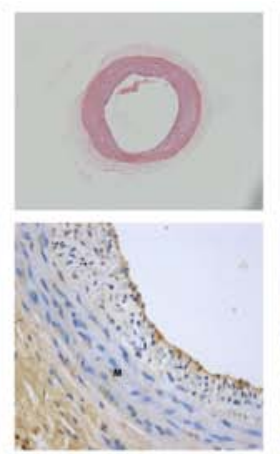

$\mathbf{D M}+\mathbf{B I}$
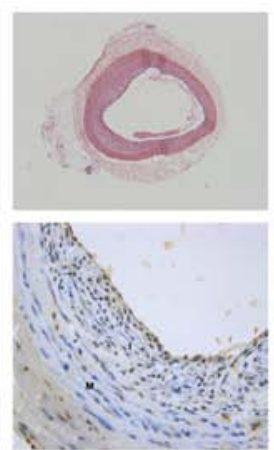

DM+BI+Ad-GFP
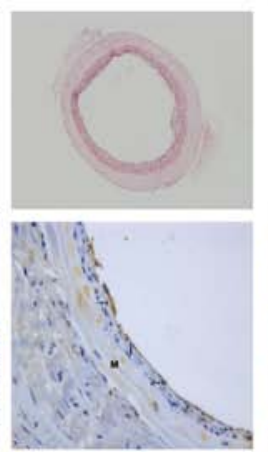

DM+BI+Ad-RNF10

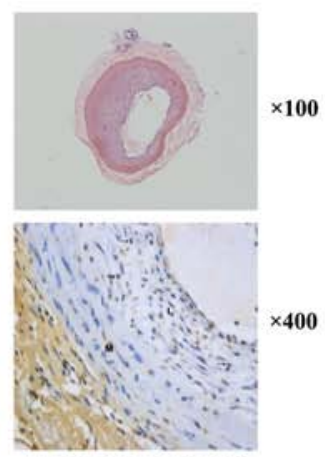

DM+BI+Ad-shRNF10
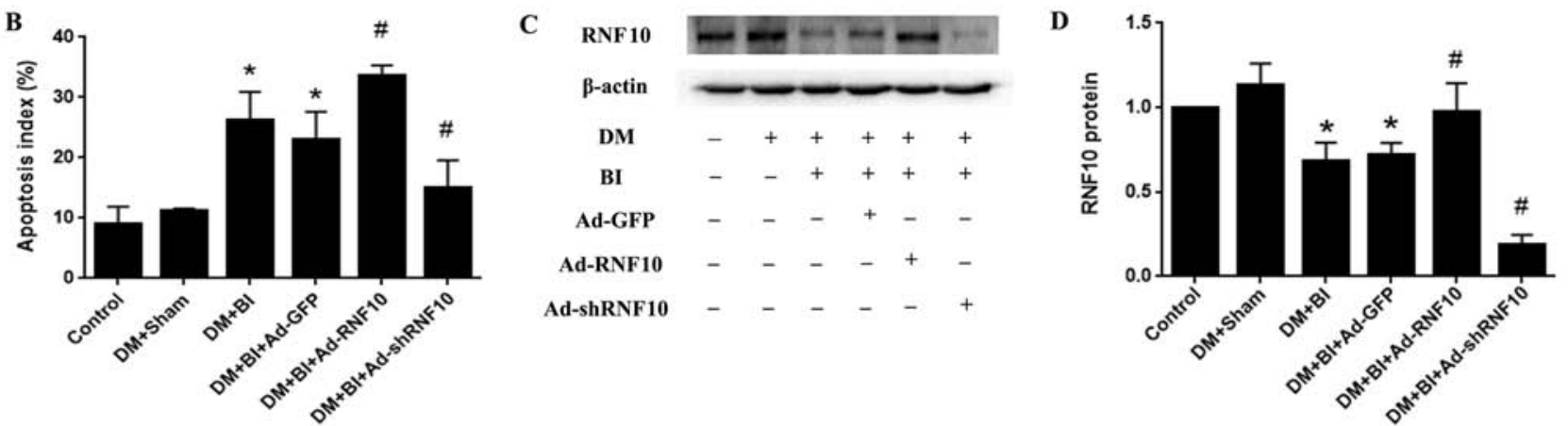

Figure 1. Effects of RNF10 on apoptosis in the carotid artery. (A) H\&E staining (original magnification, x100) (top panels) and TUNEL staining (original magnification, x400) (bottom panels) on cross-sections of the carotid arteries. (B) Quantification of the apoptosis index, defined as the ratio of TUNEL-positive cell neointimal numbers to the total neointimal numbers $(\mathrm{n} \geq 3)$. (C and D) Representative western blot analysis of RNF10 protein and quantitative analysis of RNF10 protein level ( $\geq 3$ ). ${ }^{*} \mathrm{P}<0.05$ vs. the $\mathrm{DM}+$ Sham group; ${ }^{*} \mathrm{P}<0.05$ vs. the DM+BI+Ad-GFP group. DM, diabetes mellitus; RNF10, RING finger protein 10 ; shRNF10, short hairpin RNF10; BI, balloon injury; Ad, adenovirus; GFP, green fluorescent protein; TUNEL, terminal deoxynucleotidyl transferase dUTP nick end labeling; H\&E, haematoxylin and eosin.

overnight at $4{ }^{\circ} \mathrm{C}$ with primary antibodies, including antibodies for Bcl-2 (1:2,000; cat. no. 12789; ProteinTech, Wuhan, Hubei, China), Bax (1:2,000; cat. no. 50599; ProteinTech), NF-кB (1:1,000; cat. no. 14220; ProteinTech), Cyclin D1 (1:1,000; cat. no. 60186; ProteinTech), CDK4 (1:1,000; cat. no. 11026; ProteinTech), RNF10 (1:500; cat. no. abs127972; Absin Bioscience Inc., Shanghai, China) and $\beta$-actin $(1: 5,000$; cat. no. 20536; ProteinTech). Enhanced chemiluminescence development methods (Advansta Inc., Menlo Park, CA, USA) were used to visualize the immunoblotting bands (19). The bands were analyzed with ImageLab 3.0 software (Bio-Rad Laboratories, Inc., Hercules, CA, USA).

Statistical analysis. All the data are expressed as the means \pm standard deviation (SD), and statistical analysis was performed using SPSS 19.0 (IBM Corp., Armonk, NY, USA). One-way ANOVA and LSD was used to analyse the differences among groups; $\mathrm{P}<0.05$ was considered to indicate statistical significance.

\section{Results}

Differentially expressed RNF10 affects the neointimal apoptosis index in diabetic rats. To evaluate whether vascular remodeling was induced by balloon injury, H\&E staining was performed to detect neointimal formation. In general, we observed that the balloon-injured arteries had clearly increased intimal thickness compared with that of the DM+Sham group arteries. Compared with that in the
Ad-GFP-treated group, the intimal thickness in the Ad-RNF10 group was markedly reduced. However, the intimal thickness was clearly increased in the Ad-shRNF10-treated group (Fig. 1A).

To assess whether the balloon-injured arteries were successfully transduced with the corresponding RNF10 gene, western blotting was performed to detect RNF10 protein expression. Compared with that of the arteries in the DM+Sham group, the RNF10 protein expression of the balloon-injured arteries was significantly decreased $(\mathrm{P}<0.001)$. Among the rats with balloon-injured arteries that were infected with different adenoviruses, RNF10 protein expression was significantly increased in the group receiving Ad-RNF10 $(\mathrm{P}<0.05)$ and was markedly decreased in the Ad-shRNF10 group $(\mathrm{P}<0.001)$ (Fig. 1C and D).

To examine whether RNF10 affected the apoptotic response in the model of diabetic vascular remodeling, we used TUNEL staining to determine the proportion of cells that were apoptotic in the neointima of the carotid artery. Very few apoptosis-positive cells were observed in the control group and the $\mathrm{DM}+$ Sham group, but the apoptotic index was significantly higher in the balloon-injured groups than in the DM+Sham groups $(24.34 \pm 5.15$ vs. $10.10 \pm 5.22 \%$, $\mathrm{P}<0.001)$. Compared with that in the Ad-GFP group, the proportion of apoptotic cells was significantly increased in the Ad-RNF10 group (32.52 \pm 3.77 vs. $24.18 \pm 5.56 \%, \mathrm{P}<0.05)$, but the apoptosis index was clearly decreased in the group infected with Ad-shRNF10 (14.59 \pm 4.29 vs. 24.18 $\pm 5.56 \%$, $\mathrm{P}<0.01$ ) (Fig. 1A and B). 
A
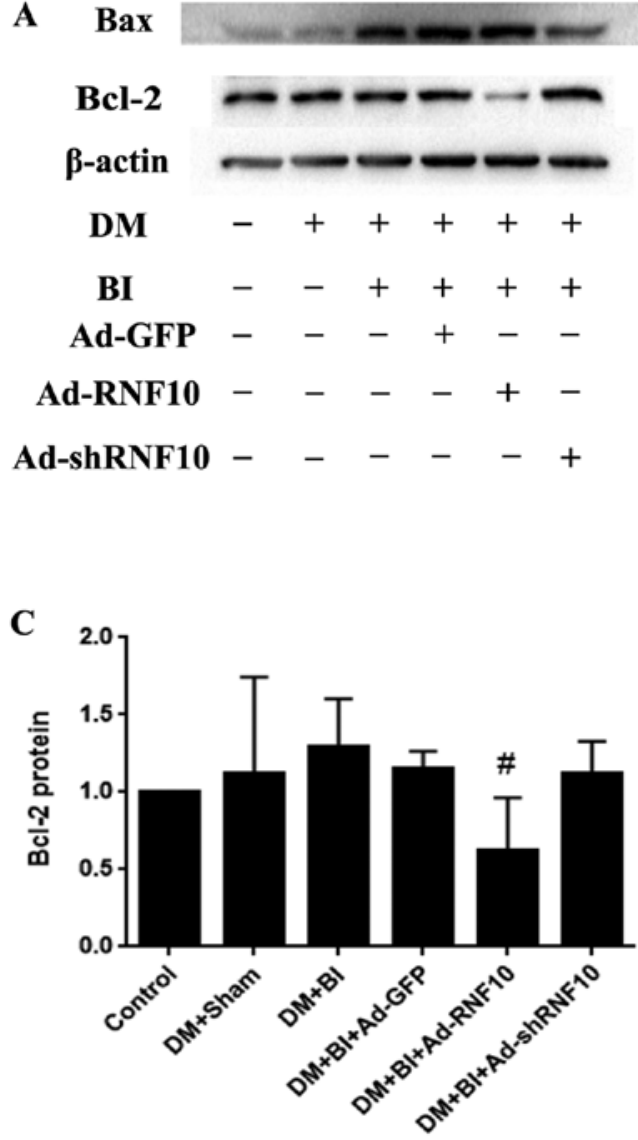

B
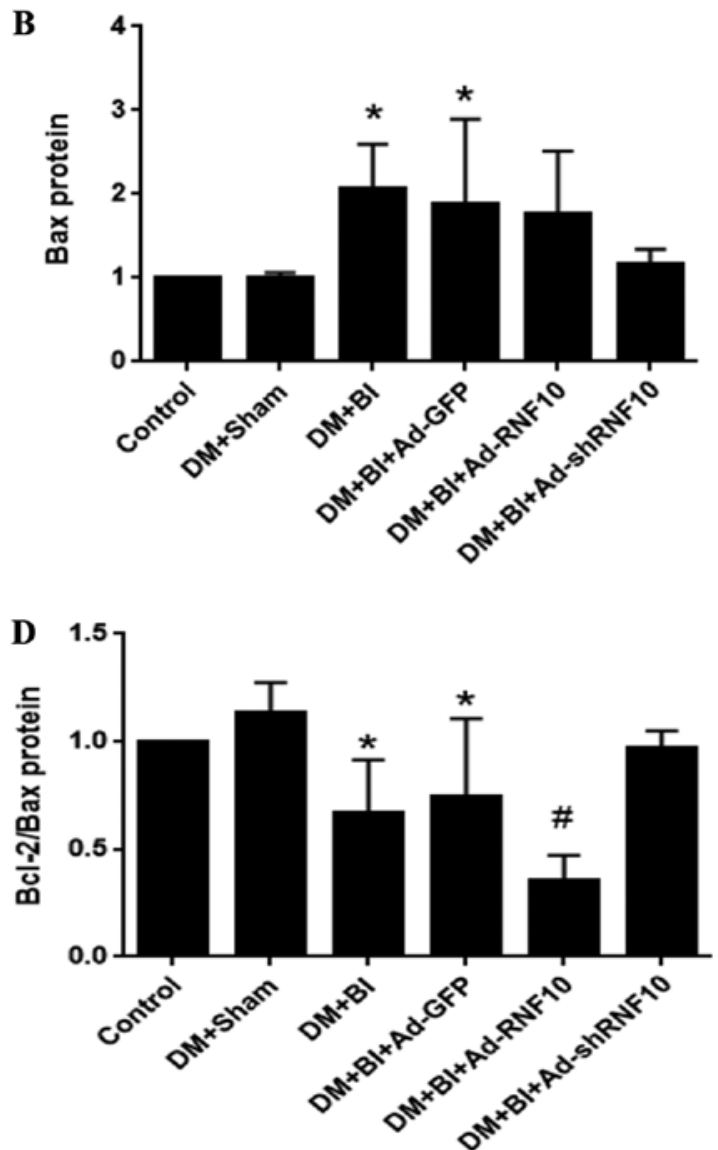

Figure 2. Effect of RNF10 on apoptotic marker proteins of Bax and Bcl-2 in the carotid artery. (A) Representative western blot analysis of Bax and Bcl-2 protein. Quantification of Bax (B), Bcl-2 (C) and Bcl-2/Bax (D) protein levels was analyzed by western blot analysis ( $\geq 3$ ). * $<<0.05$ vs. the DM+Sham group; ${ }^{\text {P }}<0.05$ vs. the DM+BI+Ad-GFP group. DM, diabetes mellitus; RNF10, RING finger protein 10; shRNF10, short hairpin RNF10; BI, balloon injury; Ad, adenovirus; GFP, green fluorescent protein; Bax, B-cell CLL/lymphoma 2 associated X protein; Bcl-2, B-cell CLL/lymphoma 2.

Overexpression of RNF10 attenuates apoptosis by decreasing the expression of marker protein Bcl-2. To verify the expression of apoptotic proteins in carotid vessels, western blotting was performed to detect the levels of the Bax and Bcl-2 proteins. Bax protein expression was significantly increased in the DM+BI group compared to the DM+Sham group $(\mathrm{P}<0.05)$, but there were no significant differences due to infection with Ad-GFP, Ad-RNF10 or Ad-shRNF10 ( $\mathrm{P}>0.05)$ (Fig. 2A and B). Compared with that in the arteries of the Ad-GFP-transduced rats, the Bcl-2 protein expression in the arteries of the group receiving Ad-RNF10 group was significantly reduced $(\mathrm{P}<0.05)$, while the expression was not significantly changed in the group infected with Ad-shRNF10. In addition, the ratio of Bcl-2 to Bax was significantly decreased in the Ad-RNF10 group $(\mathrm{P}<0.05)$ and showed a trend towards a slight increase in the group infected with Ad-shRNF10, but there was no significant difference (P>0.05) (Fig. 2A, C and D).

Overexpression of RNF10 suppresses VSMC inflammation levels by inhibiting the marker protein $N F-\kappa B$ in diabetic rats. To determine whether the RNF10 gene was successfully transduced into carotid arteries after balloon injury, qPCR was used to detect RNF10 mRNA expression. Initially, the transcription level of RNF10 in the DM +Sham group was higher than that in the control group $(\mathrm{P}<0.001)$. After balloon injury, this elevation in RNF10 mRNA was clearly reversed $(\mathrm{P}<0.001)$. Compared with that in the Ad-GFP group, the expression of RNF10 mRNA in the group receiving Ad-RNF10 was significantly enhanced $(\mathrm{P}<0.05)$, and the expression in the group receiving Ad-shRNF10 was markedly reduced $(\mathrm{P}<0.001)$ (Fig. 3A).

To detect whether the RNF10 gene was involved in the inflammatory response of vascular remodeling in diabetic rats, we evaluated the mRNA levels of the inflammatory indicators MCP-1 and TNF- $\alpha$, and analysed the expression of the inflammatory marker protein NF- $\mathrm{B}$. Compared with those in the control group, the levels of MCP-1 and TNF- $\alpha$ mRNA in the $\mathrm{DM}+$ Sham group were markedly increased $(\mathrm{P}<0.05)$. In addition, the expression of these inflammatory indicators in the $\mathrm{DM}+\mathrm{BI}$ group was higher than that in the $\mathrm{DM}+$ Sham group $(\mathrm{P}<0.05)$. Importantly, the MCP-1 and TNF- $\alpha$ transcription levels were decreased in the group receiving Ad-RNF10 compared with the group receiving Ad-GFP $(\mathrm{P}<0.001)$. Concerning the inflammatory marker protein $\mathrm{NF}-\kappa \mathrm{B}, \mathrm{NF}-\kappa \mathrm{B}$ levels were significantly higher in groups with balloon injury than in the $\mathrm{DM}+$ Sham group $(\mathrm{P}<0.05)$. Furthermore, balloon injured arteries infected with Ad-RNF10 displayed a significant reduction in $\mathrm{NF}-\kappa \mathrm{B}$ protein compared with carotid arteries infected with Ad-GFP $(\mathrm{P}<0.05)$. However, the mRNA expression of the inflammatory indicators MCP-1 and TNF- $\alpha$ mRNA, and the protein expression of NF- $\kappa \mathrm{B}$ were 

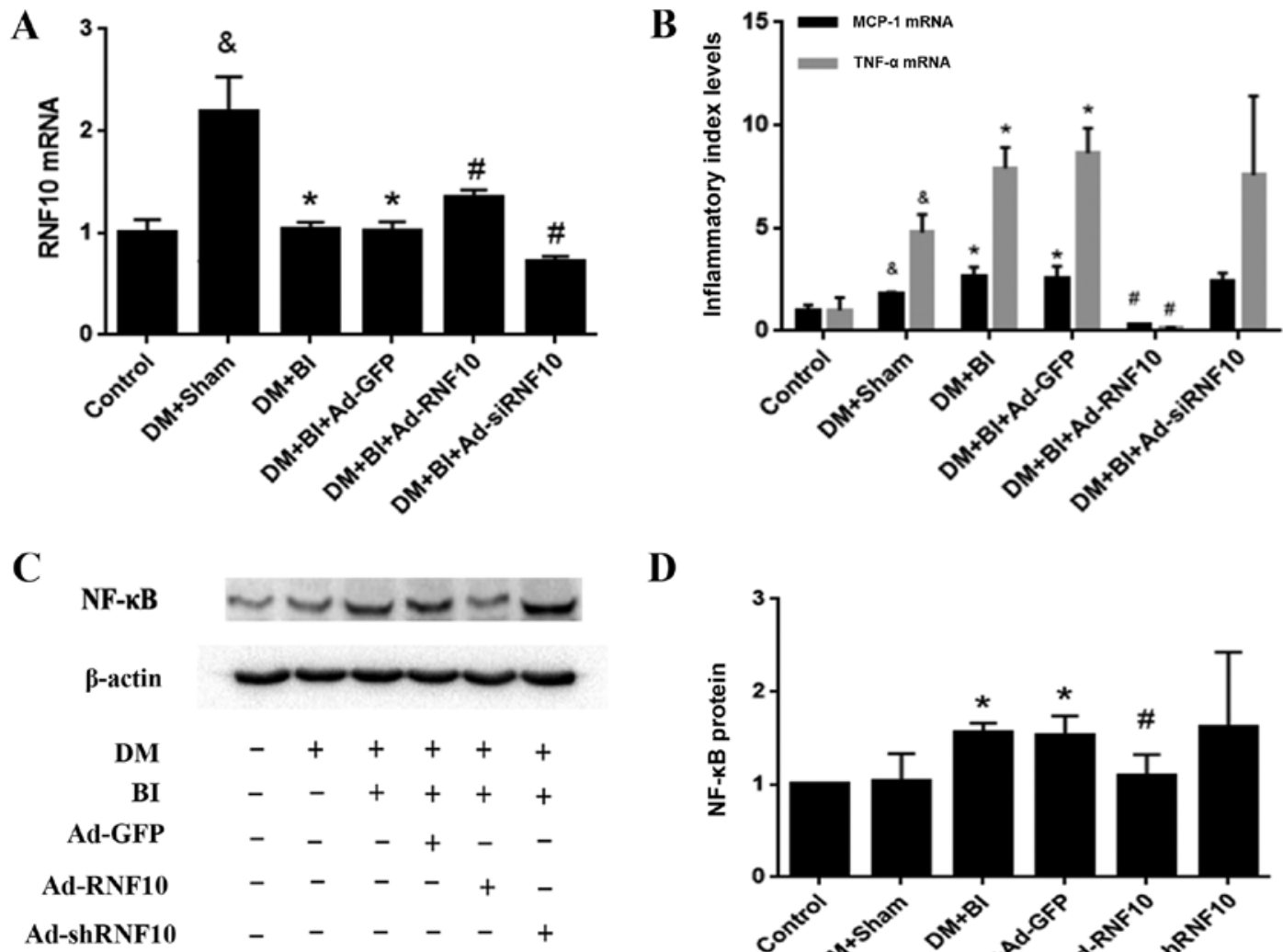

D

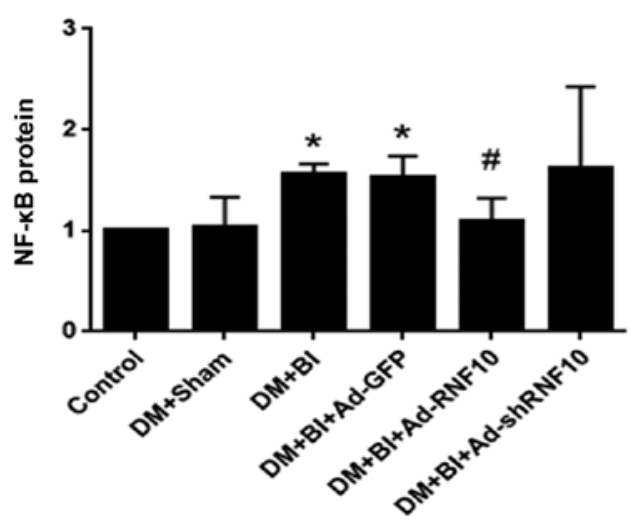

Figure 3. Effects of RNF10 on the inflammatory responses in carotid artery injury. (A) RNF10, (B) MCP-1 and TNF- $\alpha$ mRNA expression levels were analyzed with qPCR ( $\mathrm{n} \geq 3$ ). (C) Representative western blot of NF- $\kappa$ B protein. (D) Quantification of NF- $\kappa$ B protein level as determined by western blot analysis ( $\geq 3$ ). ${ }^{\circledR} \mathrm{P}<0.05$ vs. the corresponding control group; ${ }^{\mathrm{P}}<0.05$ vs. the corresponding $\mathrm{DM}+$ Sham group; ${ }^{\sharp} \mathrm{P}<0.05$ vs. the corresponding DM+BI+Ad-GFP group. DM, diabetes mellitus; RNF10, RING finger protein 10; shRNF10, RNF10 short hairpin RNA; BI, balloon injury; Ad, adenovirus; GFP, green fluorescent protein; qPCR, quantitative polymerase chain reaction; TNF- $\alpha$, tumor necrosis factor $\alpha$; MCP-1, monocyte chemotactic protein 1; NF- $\kappa \mathrm{B}$, nuclear factor- $\kappa$-gene binding.

not significantly changed by Ad-shRNF10 infection $(\mathrm{P}>0.05)$ (Fig. 3B-D).

Differentially expressed RNF10 regulates vascular hyperproliferation in diabetic rats. To assess whether RNF10 regulated the hyperproliferation of VSMCs in diabetic rats after balloon injury, we detected the protein levels of cyclin D1 and CDK4 in the carotid tissue. After balloon injury in DM rats, the protein levels of cyclin D1 and CDK4 were markedly higher than those in the $\mathrm{DM}+$ Sham group $(\mathrm{P}<0.05)$. In comparison to the Ad-GFP group, the Ad-RNF10 group showed significantly inhibited cyclin D1 protein expression $(\mathrm{P}<0.05)$. While shRNF10 slightly promoted cyclin D1 expression, there was no significant difference between the Ad-RNF10-treated group and the Ad-GFP-treated group $(\mathrm{P}>0.05)$. In addition, CDK4 protein expression was significantly decreased in the Ad-RNF10 group and significantly increased in the Ad-shRNF10 group compared with the Ad-GFP group $(\mathrm{P}<0.05)$ (Fig. 4A-C).

\section{Discussion}

In the present study, we found that overexpression of RING finger protein 10 (RNF10) promoted an apoptotic reaction, inhibited the inflammatory response and repressed vascular smooth muscle cell (VSMC) hyperproliferation in carotid arteries, all of which showed that RNF10 exerts a protective influence in diabetic vascular remodeling. In addition, shRNF10 had an opposite impact on hyperproliferation and apoptosis, further destroying arterial function and structure in diabetes. Taken together, the results of this study demonstrated that changes in RNF10 expression affected vascular remodeling, indicating that the RNF10 gene plays a central role in diabetic rats with vascular complications (Fig. 5).

A series of studies have revealed the relationship between the ubiquitin-proteasome system (UPS) and vascular remodeling, demonstrating that ubiquitin protease can inhibit vascular intimal proliferation in a dose-dependent manner, attenuate VSMC inflammation and promote apoptosis by decreasing the activity of the NF- $\kappa \mathrm{B}$ signalling pathway $(9,20)$. RNF10, an E3 ubiquitin ligase, is widely expressed in mammalian tissues and actively participates in the UPS. Previous studies have shown that RNF10 not only affects the signal transduction of insulin, but also regulates nearly all physiological functions, including apoptosis and the cell cycle $(11,21)$. Consistent with the results described above, our present study found that the level of RNF10 mRNA was elevated in the DM group, but this phenomenon was reversed following the balloon injury 
A CDK4

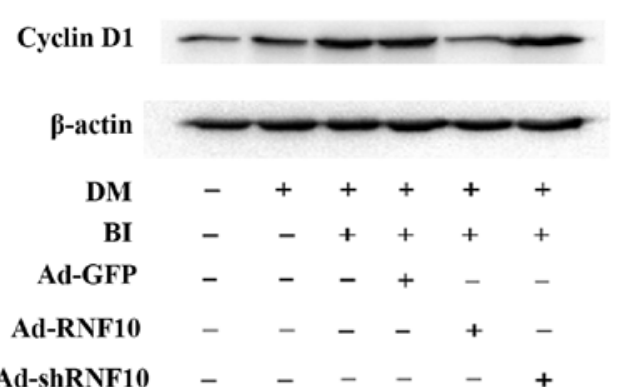

B

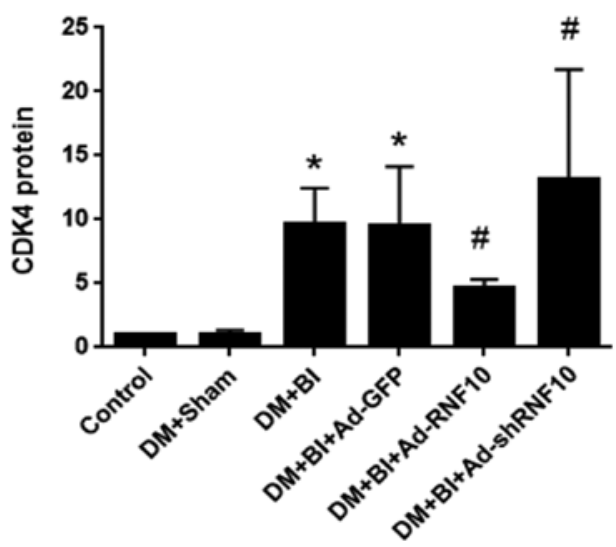

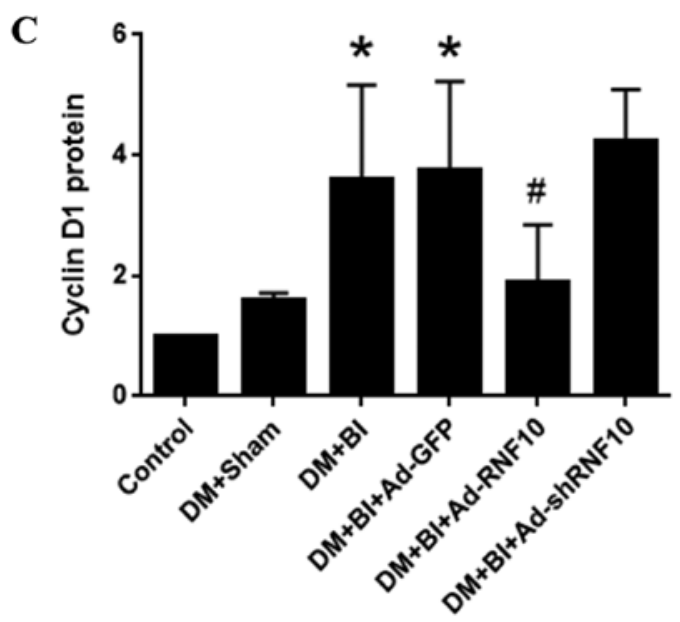

Figure 4. Effects of RNF10 on proliferation in the carotid artery. (A) Representative western blot of CDK4 and cyclin D1 protein. (B and C) Quantification of CDK4 protein and cyclin D1 levels was assessed by western blot analysis ( $\mathrm{n} \geq 3$ ). ${ }^{*} \mathrm{P}<0.05$ vs. the DM+Sham group; ${ }^{*} \mathrm{P}<0.05$ vs. DM+BI+Ad-GFP. DM, diabetes mellitus; RNF10, RING finger protein 10; shRNF10, short hairpin RNF10; BI, balloon injury; Ad, adenovirus; GFP, green fluorescent protein; CDK4, cyclin dependent kinase 4 .
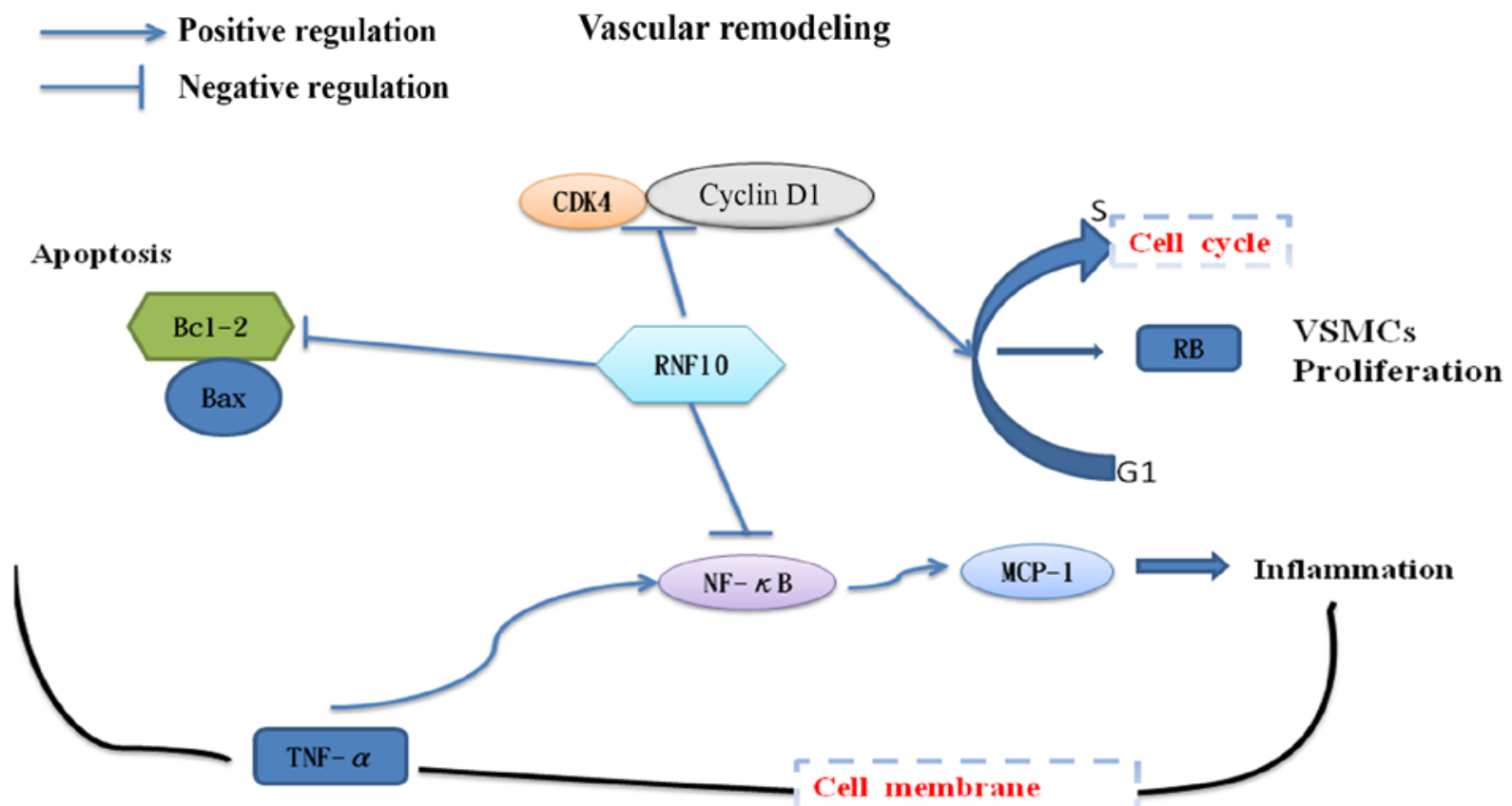

Figure 5. Effects of RNF10 on vascular remodeling in diabetic rats with carotid artery injury. RNF10 as a key factor attenuated vascular remodeling via suppressing inflammatory responses, hyperproliferation and inhibitory apoptosis. RNF10, RING finger protein 10; VSMCs, vascular smooth muscle cells;

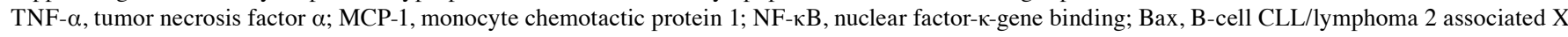
protein; Bcl-2, B-cell CLL/lymphoma 2; CDK4, cyclin dependent kinase 4. 
of carotid arteries. This finding indicated that RNF10 may be inhibited after balloon injury and may function in diabetic vascular remodeling. Overall, this study further investigated the comprehensive effects of RNF10 on vascular remodeling in a diabetic model, including the role of RNF10 in anti-inflammation, anti-proliferation and pro-apoptosis reactions.

Many clinical trials have demonstrated that chronic hyperglycaemia is a pathological condition that accelerates the development of multiple cardiovascular diseases and is clearly correlated with microvascular and macrovascular complications in diabetes (22). In diabetic patients, hyperglycaemia-induced activation of the NF- $\kappa \mathrm{B}$ signalling pathway causes the overproduction of a large number of cytokines, which is a crucial aspect of the process of diabetic vascular remodeling. These activated cytokines, such as TNF- $\alpha$ and MCP-1, trigger the aggregation and infiltration of inflammatory cells, affecting the permeability and structure of blood vessels, and contributing to the development of diabetic vascular disease (23-26). Our study confirmed that overexpression of RNF10 might inhibit the mRNA expression of the inflammatory markers TNF- $\alpha$ and MCP-1 via the NF- $\kappa$ B signalling pathway. However, shRNF10 could not change the levels of these inflammatory indicators, probably due to compensatory mechanisms in the organisms. Therefore, RNF10 might be a crucial target to regulate inflammation reactions in diabetic vascular remodeling.

VSMC hyperproliferation is also an important part of the vascular remodeling process and is affected by the cell cycle regulatory proteins cyclin D1 and CDK4. The regulation of the cell cycle depends on complexes of cyclin and CDK, which are core materials driving the smooth progression of the cell cycle $(27,28)$. A previous study demonstrated that RNF10 could cause a 1.95 -fold activation of the $\mathrm{P} 21$ promoter. $\mathrm{P} 21$, an important cell cycle regulatory protein, effectively inhibits $\mathrm{pRb}$ phosphorylation mediated by the cyclin D1-CDK4 complex, thereby slowing the cell cycle transition from the G1 phase to the $\mathrm{S}$ phase $(21,29)$. Based on these findings, we suggest that the inhibition of VSMC hyperproliferation may be ascribed to the modulation of P21 expression and the cyclin D1-CDK4 complex. Furthermore, shRNF10 also significantly aggravated hyperproliferation by increasing CDK4 protein levels. However, as a result of insufficient sample size, the levels of cyclin D1 were not significantly altered. Therefore, RNF10 might be a central factor that regulates VSMC hyperproliferation in diabetes.

Furthermore, the apoptosis reaction is also involved in diabetic rats with microvascular and macrovascular complications. The inhibition of apoptosis in VSMCs exposed to high glucose determines the proliferation level in vascular remodeling and is also regulated by the anti-apoptosis gene Bcl-2 and the pro-apoptosis gene Bax (5). Both Bcl-2 and Bax are crucial members of the Bcl-2 family that widely regulate apoptosis, and their ratio always reflects the apoptosis level in cells (30). In our previous study, we observed that RNF10 promoted VSMC apoptosis and downregulated the anti-apoptotic $\mathrm{Bcl}-2$ protein to prevent neointimal hyperplasia in metabolic syndrome (14). Although the $\mathrm{Bcl}-2 / \mathrm{Bax}$ protein ratio was slightly increased without a significant difference, the increase may have resulted from insufficient sample size. In this study, overexpression of RNF10 was found to promote apoptosis to prevent vascular remodeling by decreasing the $\mathrm{Bcl}-2 / \mathrm{Bax}$ ratio, and shRNF10 was also found to inhibit the apoptosis response to aggravate neointimal formation in diabetic rats. Therefore, RNF10 may be considered a key factor in regulating apoptosis in vascular remodeling in diabetic rats.

In summary, RNF10 may be the central target for the regulation of diabetic vascular remodeling due to its pro-apoptosis, anti-inflammation and anti-hyperproliferation activity (Fig. 5). However, the specific mechanism of the signalling pathway remains unclear and will be investigated in future studies.

\section{Acknowledgements}

The authors would like to thank The First Affiliated Hospital of Chongqing Medical University for the experimental platform assistance.

\section{Funding}

This study was sponsored by the National Natural Science Foundation of China (grant no. 31501097).

\section{Availability of data and materials}

The datasets used and/or analysed during the current study are available from the corresponding author on reasonable request.

\section{Authors' contributions}

SL, GY, WH and RW performed the histological examination and other animal experiments. SL and MC analyzed and interpreted all the data. PP was a major contributor in designing the study and writing the manuscript. All authors read and approved the final manuscript and agree to be accountable for all aspects of the research in ensuring that the accuracy or integrity of any part of the work are appropriately investigated and resolved.

\section{Ethics approval and consent to participate}

All animal experiments were approved by the Ethics Committee of Chongqing Medical University (Chongqing, China) and were performed according to the ARRIVE guidelines and the UK Animals (Science Procedures) Act, 1986 (https://assets.publishing.service.gov.uk/government/ uploads/system/uploads/attachment_data/file/116843/aspadraft-guidance.pdf), and its associated guidelines.

\section{Patient consent for publication}

Not applicable.

\section{Competing interests}

The authors declare that there have no competing interests.

\section{References}

1. Guariguata L, Whiting DR, Hambleton I, Beagley J, Linnenkamp U and Shaw JE: Global estimates of diabetes prevalence for 2013 and projections for 2035. Diabetes Res Clin Pract 103: 137-149, 2014. 
2. Doran AC, Meller N and McNamara CA: Role of smooth muscle cells in the initiation and early progression of atherosclerosis. Arterioscler Thromb Vasc Biol 28: 812-819, 2008.

3. Huang CN, Chan KC, Lin WT, Su SL and Peng CH: Hibiscus sabdariffa inhibits vascular smooth muscle cell proliferation and migration induced by high glucose-a mechanism involves connective tissue growth factor signals. J Agric Food Chem 57: 3073-3079, 2009.

4. Hall JL, Matter CM, Wang X and Gibbons GH: Hyperglycemia inhibits vascular smooth muscle cell apoptosis through a protein kinase C-dependent pathway. Circ Res 87: 574-580, 2000.

5. Li H, Télémaque S, Miller RE and Marsh JD: High glucose inhibits apoptosis induced by serum deprivation in vascular smooth muscle cells via upregulation of Bcl-2 and Bcl-xl. Diabetes 54: 540-545, 2005.

6. Balasubramanyam M, Sampathkumar R and Mohan V: Is insulin signaling molecules misguided in diabetes for ubiquitin-proteasome mediated degradation? Mol Cell Biochem 275: 117-125, 2005.

7. Patterson C: Search and destroy: The role of protein quality control in maintaining cardiac function. J Mol Cell Cardio 40 438-441, 2006

8. Drews O and Taegtmeyer H: Targeting the ubiquitin-proteasome system in heart disease: The basis for new therapeutic strategies Antioxid Redox Signal 21: 2322-2343, 2014.

9. Meiners S, Laule M, Rother W, Guenther C, Prauka I, Muschick P, Baumann G, Kloetzel PM and Stangl K: Ubiquitin-proteasome pathway as a new target for the prevention of restenosis. Circulation 105: 483-489, 2002.

10. Herrmann J, Ciechanover A, Lerman LO and Lerman A: The ubiquitin-proteasome system in cardiovascular diseases-a hypothesis extended. Cardiovasc Res 61: 11-21, 2004.

11. Seki N, Hattori A, Sugano S, Muramatsu M and Saito T: cDNA cloning, expression profile, and genomic structure of human and mouse RNF10/Rnf 10 genes, encoding a novel RING finger protein. J Hum Genet 45: 38-42, 2000.

12. Saurin AJ, Borden KL, Boddy MN and Freemont PS: Does this have a familiar RING? Trends Biochem Sci 21: 208-214, 1996.

13. Huang K, Nair AK, Muller YL, Piaggi P, Bian L, Del Rosario M, Knowler WC, Kobes S, Hanson RL, Bogardus C and Baier LJ: Whole exome sequencing identifies variation in CYB5A and RNF10 associated with adiposity and type 2 diabetes. Obesity (Silver Spring) 22: 984-988, 2014

14. Yu G, Chen J, Li S, Pu P, Huang W, Zhao Y, Wang R and Lei H: RING finger protein 10 prevents neointimal hyperplasia by promoting apoptosis in vitro and in vivo. Life Sci 208: 325-332, 2018.

15. Yang J, Fan Z, Yang J, Ding J, Yang C and Chen L: MicroRNA-24 attenuates neointimal hyperplasia in the diabetic rat carotid artery injury model by inhibiting Wnt 4 signaling pathway. Int $\mathrm{J}$ Mol Sci 17: pii: E765, 2016.

16. Yuan X, Zhang T, Yao F, Liao Y, Liu F, Ren Z, Han L, Diao L, Li Y, Zhou B, et al: THO complex-dependent posttranscriptional control contributes to vascular smooth muscle cell fate decision. Circ Res 123: 538-549, 2018.
17. Zhang Y, Xia G, Zhang Y, Liu J, Liu X, Li W, Lv Y, Wei S, Liu J and Quan J: Palmitate induces VSMC apoptosis via toll like receptor (TLR)4/ROS/p53 pathway. Atherosclerosis 263: 74-81, 2017.

18. Yang D, Sun C, Zhang J, Lin S, Zhao L, Wang L, Lin R, Lv J and Xin S: Proliferation of vascular smooth muscle cells under inflammation is regulated by NF- $\mathrm{B}$ p65/microRNA-17/RB pathway activation. Int $\mathrm{J}$ Mol Med 41: 43-50, 2018.

19. Pan CH, Li PC, Chien YC, Yeh WT, Liaw CC, Sheu MJ and $\mathrm{Wu} \mathrm{CH}$ : Suppressive activities and mechanisms of ugonin J on vascular smooth muscle cells and balloon angioplasty-induced neointimal hyperplasia. Phytother Res 32: 312-320, 2018.

20. Lecker SH, Solomon V, Price SR, Kwon YT, Mitch WE and Goldberg AL: Ubiquitin conjugation by the $\mathrm{N}$-end rule pathway and mRNAs for its components increase in muscles of diabetic rats. J Clin Invest 104: 1411-1420, 1999.

21. Lin J, Friesen MT, Bocangel P, Cheung D, Rawszer K and Wigle JT: Characterization of mesenchyme homeobox 2 (MEOX2) transcription factor binding to RING finger protein 10 Mol Cell Biochem 275: 75-84, 2005.

22. Paolisso G, Rizzo MR, Barbieri M, Manzella D, Ragno E and Maugeri D: Cardiovascular risk in type 2 diabetics and pharmacological regulation of mealtime glucose excursions. Diabetes Metab 29: 335-340, 2003

23. Navarro-González Juan $F$ and Mora-Fernández C: The role of inflammatory cytokines in diabetic nephropathy. J Am Soc Nephrol 19: 433-442, 2008.

24. Winkler G, Salamon F, Harmos G, Salamon D, Speer G, Szekers O, Hajós P, Kovács M, Simon K and Cseh K: Elevated serum tumor necrosis factor-alpha concentrations and bioactivity in type 2 diabetics and patients with android type obesity. Diabetes Res Clin Pract 42: 169-174, 1998.

25. Bayón Y, Alonso A, Hernández M, Nieto ML and Sánchez Crespo M: Mechanisms of cell signaling in immune-mediated inflammation. Cytokines Cell Mol Ther 4: 275-286, 1998.

26. Brownlee M: The pathobiology of diabetic complications: A unifying mechanism. Diabetes 54: 1615-1625, 2005.

27. Sherr CJ and Roberts JM: CDK inhibitors: Positive and negative regulators of G1-phase progression. Genes Dev 13: 1501-1512, 1999.

28. Sylvester AM, Chen D, Krasinski K and Andrés V: Role of c-fos and $\mathrm{E} 2 \mathrm{~F}$ in the induction of cyclin A transcription and vascular smooth muscle cell proliferation. J Clin Invest 101: 940-948, 1998.

29. Lu $\mathrm{Z}$ and Hunter T: Ubiquitylation and proteasomal degradation of the p21(Cip1), p27(Kip1) and p57(Kip2) CDK inhibitors. Cell Cycle 9: 2342-2352, 2010.

30. Korsmeyer SJ: BCL-2 gene family and the regulation of programmed cell death. Cancer Res 59 (Suppl 7): S1693-S1700, 1999.

(i) $($ This work is licensed under a Creative Common Attribution-NonCommercial-NoDerivatives 4.0 International (CC BY-NC-ND 4.0) License. 